\title{
Predictive value for progression to tuberculosis by IGRA and TST in immigrant
} contacts

\author{
S.V. Kik*\#, W.P.J. Franken ${ }^{\star}$, M. Mensen+, F.G.J. Cobelens*,\#, M. Kamphorst ${ }^{\S}$, \\ S.M. Arend", C. Erkens", A. Gebhard*, ${ }^{\star}$, M.W. Borgdorff*,\# and S. Verver, ${ }^{\star}$
}

ABSTRACT: The authors determined the positive predictive value (PPV) for progression to tuberculosis (TB) of two interferon- $\gamma$ release assays (IGRAs), QuantiFERON-TB ${ }_{\mathbb{B}}$ Gold In-tube (QFT-GIT) and T-SPOT.TB ${ }_{\mathbb{B}}$, and the tuberculin skin test (TST) in immigrants contacts.

Immigrant close contacts of sputum smear-positive TB patients were included when aged $\geqslant 16 \mathrm{yrs}$ and their TST result was $\geqslant 5 \mathrm{~mm} 0$ or 3 months after diagnosis of the index patient. Contacts were followed for the next 2 yrs for development of TB disease.

Of 339 immigrant contacts with TST $\geqslant 5 \mathrm{~mm}, 324$ and 299 had valid results of QFT-GIT and T-SPOT.TB ${ }^{\circledR}$, respectively. Nine contacts developed active TB. One patient had not been tested with TST, while another patient had not been tested with QFT-GIT and T-SPOT.TB. The PPV for progression to TB during this period was $9 / 288=3.1 \%(95 \% \mathrm{Cl} 1.3-5.0 \%)$ for TST $\geqslant 10 \mathrm{~mm}, 7 /$ $184=3.8 \%(95 \% \mathrm{Cl} 1.7-5.9 \%)$ for TST $\geqslant 15 \mathrm{~mm}, 5 / 178=2.8 \%$ (95\% Cl 1.0-4.6\%) for QFT-GIT and 6/ $181=3.3 \%(95 \%$ Cl $1.3-5.3 \%)$ for T-SPOT.TB. Sensitivity was $100 \%, 88 \%, 63 \%$ and $75 \%$, respectively.

The predictive values of QFT-GIT, T-SPOT.TB ${ }_{\mathbb{B}}$ and TST for progression to TB disease among immigrant close contacts were comparable.

KEYWORDS: Contact tracing, immigrants, interferon- $\gamma$ release assay, predictive value, tuberculin skin test, tuberculosis

西 nterferon- $\gamma$ release assays (IGRAs) have emerged as an alternative for the tuberculin skin test (TST) for the diagnosis of a latent tuberculosis (TB) infection (LTBI). Currently, two commercial IGRAs are available: QuantiFERON$\mathrm{TB}_{\mathbb{B}}$ Gold In-tube (QFT-GIT; Cellesis, Carnegie, Australia) and T-SPOT.TB (Oxford Immunotec, Abingdon, UK). These IGRAs measure the immune response to Mycobacterium tuberculosisspecific antigens. IGRA results are not affected by previous bacille Calmette-Guérin (BCG) vaccination and most infections of nontuberculous mycobacteria [1]. Furthermore, repeated testing does not influence later test results, in contrast to the boosting effect that can be observed when the TST is repeated over time [2]. Several countries incorporated the IGRA as a diagnostic test for LTBI in their guidelines and recommend its use as a confirmative test after a positive TST $[3,4]$ or as an alternative to the TST [4-6]. However, more direct evidence from studies with follow-up of untreated latently infected subjects would lend scientific support to the implementation of these guidelines $[1,7]$.
So far, few prospective studies assessed progression of TB among contacts of infectious pulmonary TB patients in relation to IGRA results [8-12]. While one study showed that the QFT-GIT was a more accurate indicator for progression to active disease than the TST at a cut-off of $5 \mathrm{~mm}$ [9], two other studies found that the in-house enzymelinked immunosorbent spot (ELISPOT) assay and TST both missed some of the contacts who progressed to TB disease. It is unclear if these different outcomes can be attributed to the different IGRA used, the type of contacts included in these studies, or to differences in the infection prevalence.

In the current study we assessed the positive predictive value (PPV) for TB disease of QFTGIT, T-SPOT.TB ${ }_{\circledR}$ and TST in immigrant individuals in the Netherlands who were recently exposed to infectious pulmonary TB patients. To our knowledge, this is the first longitudinal study that describes the predictive value of both commercially available IGRAs in a population with high risk of recent infection, a high lifetime
AFFILIATIONS

*KNCV Tuberculosis Foundation, The Hague,

${ }^{\#}$ Center for Infection and Immunity

Amsterdam, Academic Medical

Center, Amsterdam,

+Dept of Tuberculosis Control,

Municipal Health Service,

Amsterdam,

"Dept of Infectious Diseases, Leiden University Medical Center, Leiden,

${ }^{\S}$ Dept of Tuberculosis Control,

Municipal Public Health Service

Rotterdam-Rijnmond, Rotterdam, and

${ }^{f}$ Dept of Tuberculosis Control,

Municipal Health Service West-

Brabant, Breda, The Netherlands.

CORRESPONDENCE

S.V. Kik

KNCV Tuberculosis Foundation

P0 Box 146

2501 CC

The Hague

The Netherlands

E-mail: kiks@kncvtbc.nl

Received:

June 232009

Accepted after revision:

Sept 182009

First published online:

Oct 192009

European Respiratory Journal

Print ISSN 0903-1936

Online ISSN 1399-3003 
risk of previous infection and a low risk of re-infection after inclusion.

\section{MATERIAL AND METHODS Study subjects}

Between April 2005 and July 2007, close contacts of sputum smear-positive pulmonary TB patients when aged $\geqslant 16 \mathrm{yrs}$ and born in a TB endemic country (see Appendix) were recruited shortly after the diagnosis of the index patient. Furthermore, we included Dutch-born individuals when at least one of their parents was born in a TB endemic country and they were BCG vaccinated, since their TST results may be false-positive owing to their BCG status. Recruitment took place at 15 municipal health services (MHSs) throughout the Netherlands. We excluded contacts with known conditions associated with an increased risk of progression to disease (including diabetes and HIV infection) and individuals who were given preventive treatment.

\section{Data collection}

Screening of close contacts in a contact investigation is performed in two rounds in the Netherlands, first shortly after the diagnosis of the index patient and secondly 812 weeks later. At the time of recruitment all contacts underwent chest radiography to exclude the presence of active TB disease. Additionally, a TST was administered (two tuberculin units, purified protein derivative RT23 in Tween-80; Statens Serum Institute, Copenhagen, Denmark) and read after 48$72 \mathrm{~h}$. Contacts with TST results $\geqslant 5 \mathrm{~mm}$ were interviewed and blood was obtained for T-SPOT.TB ${ }$ and QFT-GIT. If TST was $<5 \mathrm{~mm}$ in the first round it was repeated at the second round and only followed by IGRA testing if $\geqslant 5 \mathrm{~mm}$. Individuals who underwent their first TST during the second round of the contact investigation were tested once. Known past TST responders (TST $\geqslant 10 \mathrm{~mm}$ ) did not undergo TST testing, but were immediately tested with IGRA. Characteristics of the cohort and factors related to positive test outcomes are described elsewhere [13].

Contacts with TST results $\geqslant 5 \mathrm{~mm}$ were invited for follow-up visits at 6, 12, 18 and 24 months after inclusion and were interviewed and investigated for signs and symptoms suggestive of TB disease. Contacts who did not show up for their follow-up visit after several invitations were, if possible, interviewed by telephone.

\section{Ethics}

Ethical approval for this study was obtained from the Netherlands Central Committee on Research Involving Human Subjects (CCMO, P04.1214C) and all participants provided oral and written informed consent. Contacts with possible LTBI in our study did not received preventive treatment, in accordance with the common practice in the Netherlands. The justification for this policy is that among adults with a high likelihood of remote (instead of recent) infection and the possibility of false-positive TST results due to previous BCG vaccination the benefit of preventive therapy may not outweigh the risks related to the chemotherapy.

\section{Incident TB cases}

Contacts diagnosed with $\mathrm{TB} \geqslant 3$ months after the diagnosis of the index patient were considered to be incident cases, whereas
TB cases diagnosed within the first 3 months after the diagnosis of the index patient were considered to be coprevalent and excluded from the analysis. The diagnosis of TB disease was based on chest radiography, symptoms, smear and/or culture results.

\section{Laboratory procedures}

Both IGRAs were performed according to the instructions of the manufacturers [14, 15] and tested in a single laboratory (Leiden University Medical Center, Leiden, the Netherlands), as described earlier [13]. For QFT-GIT (two-tube format) a positive test was defined as $\geqslant 0.35 \mathrm{IU} \cdot \mathrm{mL}^{-1}$. Interpretation of T-SPOT.TB $B_{\mathbb{R}}$ results was according to the latest criteria defined by the manufacturer.

When available, M. tuberculosis isolates from the incident cases and their index patients were subjected to IS6110 restriction fragment length polymorphism (RFLP) typing [16] and, if less than five bands, additionally subtyped using the polymorphic GC-rich sequence as a probe [17], to determine if the RFLP patterns were identical. Molecular typing was done at the National Institute of Public Health and the Environment (Bilthoven, the Netherlands).

\section{Predictive values}

In our primary analysis we determined the PPV, negative predictive value (NPV), sensitivity and specificity of the different tests among our cohort of contacts who, by definition, had a TST $\geqslant 5 \mathrm{~mm}$. Development of active TB was used as the "disease outcome" in these calculations; thus we determined sensitivity and specificity of the test result for progression to TB disease. The PPV was calculated as: number of incident TB cases with a positive test outcome/total number of contacts with a positive test outcome. Since the cumulative number of TB cases, and therefore the PPV, is dependent on the duration of follow-up and not all of our contacts could be followed for 2 yrs, we performed a secondary analysis to determine test parameters for progression to disease within the first 12 months of follow-up. Not all contacts attended the followup visits. Therefore, we performed an even more strict sensitivity analysis in which we determined the test parameters for disease progression among contacts who attended the follow-up up for $\geqslant 12$ months.

\section{Follow-up time}

The date of start of follow-up was defined as 3 months after the diagnosis of the index patient, or the date of blood collection for those who had IGRA testing $>3$ months after the diagnosis of their index patient. Follow-up time was calculated from the start of follow-up up to 24 months, the date of TB diagnosis, or the date of death or emigration out of the Netherlands, whichever occurred first.

To ascertain that we did not miss any incident cases, we performed a search in the Netherlands Tuberculosis Register (NTR) and assessed if any of the included contacts was registered with TB up to August 1, 2008. Since the NTR is an anonymous register, the search was based on the date of birth, sex and country of birth, and MHSs were asked to confirm if the matches between the study database and the NTR database were indeed the same person. Although we excluded contacts with TST $<5 \mathrm{~mm}$ from follow-up, the same search strategy in 
the NTR was performed to assess if any of them was registered with TB afterwards.

\section{Statistical analysis}

Poisson regression was used to estimate incidence rates and $95 \%$ confidence intervals for progression to TB per 1,000 person-yrs. For the primary analysis we constructed Kaplan-Meier curves. The equality of the survival distributions were compared by the Gehan-Breslow-Wilcoxon test that weighs the time points by the number of cases. Statistical analyses were conducted using SPSS version 16.0 for Windows (SPSS Inc., Chicago, IL, USA).

\section{RESULTS}

\section{Participants and test results}

During the study period, 380 contact investigations were conducted at the participating MHSs. Of 812 immigrant close contacts aged $\geqslant 16$ yrs, 433 (53\%) fulfilled the inclusion criteria and gave informed consent (fig. 1). Details on the comparison between contacts who were included and those who were not asked or refused participation are described elsewhere [13]. Out of $433,339(78 \%)$ contacts were eligible for follow-up since they either had TST results $\geqslant 5 \mathrm{~mm}(\mathrm{n}=322)$ or were known positive TST responders in the past $(\mathrm{n}=17)$. TST results were $\geqslant 10 \mathrm{~mm}$ in 288 out of $339(85 \%)$, and $\geqslant 15 \mathrm{~mm}$ in 184 out of $322(57 \%)$. Blood collection for IGRAs failed in 12 contacts. At recruitment, 178 (54\%) of 327 remaining individuals had a positive QFT-GIT result. For 28 individuals, no valid T-SPOT.TB $B_{\mathbb{R}}$ result was available owing to insufficient blood collection $(n=19)$, inconclusive test result $(n=5)$ or technical failure $(n=4)$. T-SPOT.TB was positive in 181 (61\%) of the remaining 299 individuals. Characteristics of the study population are given in table 1 .

\section{Incident cases}

Nine contacts developed $\mathrm{TB}$ disease $>3$ months after the diagnosis of the index patient. All were registered in the NTR and had been BCG vaccinated (table 2). None of the participants with TST $<5 \mathrm{~mm}$ and none of the participants who did not attend all follow-up visits matched with any of the TB-cases notified in the NTR. One incident case was not tested with TST at recruitment and, in another incident case, blood collection for IGRA had failed. All eight IGRA-tested patients had TST results $\geqslant 10 \mathrm{~mm}$ and seven $(88 \%)$ had results $\geqslant 15 \mathrm{~mm}$. T-SPOT.TB $B_{\mathbb{R}}$ was positive in six out eight $(75 \%)$ (all $>30$ spots), while QFT-GIT was positive in five out of eight $(63 \%)$ TB patients (four out of five were $>10 \mathrm{IU} \cdot \mathrm{mL}^{-1}$ ). The two patients with negative T-SPOT.TB $B_{\circledR}$ results were also negative in the QFT-GIT. Six patients (including all three TST-positive/ IGRA-negative) were confirmed by culture, and RFLP fingerprinting of the isolates of these six patients were identical to those of the corresponding index case.

None of the three incident patients with at least one negative IGRA result at recruitment were known to be HIV positive or

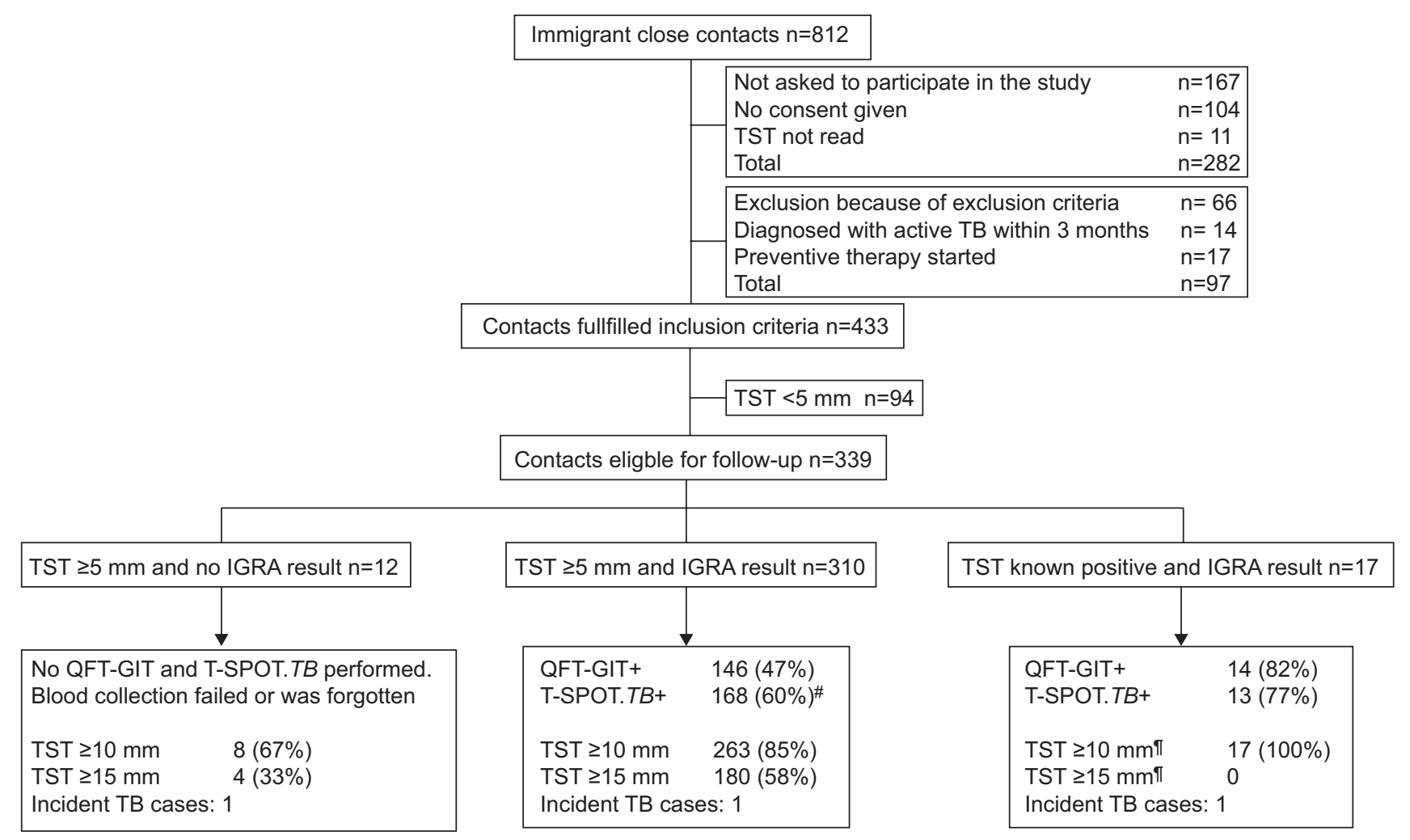

FIGURE 1. Cohort profile of recruited contacts. TST: tuberculin skin test; TB: tuberculosis; IGRA: interferon- $\gamma$ release assay; QFT-GIT: QuantiFERON TB® Gold In-tube. \#: no T-SPOT.TB result of 28 individuals because of technical failure $(n=4)$, inconclusive test results $(n=5)$ or insufficient blood collected to perform the test ( $n=19$ );

?: known positive TST results were all considered to be $\geqslant 10 \mathrm{~mm}$ (the regular cut-off for a positive TST result in the Netherlands), but were excluded from the analysis that used $15 \mathrm{~mm}$ as a cut-off since no exact indurations were known. QFT-GIT is manufactured by Cellesis, Carnegie, Australia; T-SPOT.TBß is manufactured by Oxford Immunotec, Abingdon, UK. 
to have any other immune suppressive disorder. Furthermore, none of them reported to have travelled to a TB endemic country or have been exposed to another TB case in the period between their inclusion and diagnosis. All had IGRA results far below the threshold of a positive test (QFT-GIT results: $-0.24,0.02$ and $0.04 \mathrm{IU} \cdot \mathrm{mL}^{-1}$; T-SPOT.TB® results: 0 and $1 \mathrm{spot}$ ). Contacts were tested with IGRA 4-200 days after the diagnosis of the index case (median (interquartile range) 37 (15117) days). The three contacts who developed TB and who

\begin{tabular}{|c|c|c|}
\hline TABLE 1 & \multicolumn{2}{|c|}{$\begin{array}{l}\text { Description of the study population; immigrant } \\
\text { close contacts with a tuberculin skin test (TST) } \\
\text { result } \geqslant 5 \mathrm{~mm}\end{array}$} \\
\hline Total & & 339 (100) \\
\hline \multicolumn{3}{|l|}{ Sex } \\
\hline Male & & $189(55.8)$ \\
\hline Female & & $147(43.4)$ \\
\hline Unknown & & $3(0.9)$ \\
\hline \multicolumn{3}{|l|}{ Age yrs } \\
\hline $16-24$ & & $53(15.6)$ \\
\hline $25-34$ & & 80 (23.6) \\
\hline $35-44$ & & $115(33.9)$ \\
\hline$\geqslant 45$ & & $91(26.8)$ \\
\hline \multicolumn{3}{|c|}{ Continent of birth } \\
\hline Europe, No & America & $27(8.0)$ \\
\hline South Ame & & $27(8.0)$ \\
\hline Asia & & $123(36.3)$ \\
\hline Other Africa & & $98(28.9)$ \\
\hline Sub-Sahara & Africa & $59(17.4)$ \\
\hline Unknown & & $5(1.5)$ \\
\hline \multicolumn{3}{|c|}{ Recent close contact } \\
\hline Non-house & ld contact & 185 (54.6) \\
\hline Household & ontact & 115 (33.9) \\
\hline Unknown & & 39 (11.5) \\
\hline \multicolumn{3}{|l|}{ BCG scar } \\
\hline Yes & & $274(80.8)$ \\
\hline No & & $43(12.7)$ \\
\hline Unknown & & $22(6.5)$ \\
\hline \multicolumn{3}{|c|}{ QFT-GIT result } \\
\hline Negative & & $149(44.0)$ \\
\hline Positive & & 178 (52.5) \\
\hline Not done & & $12(3.5)$ \\
\hline \multicolumn{3}{|c|}{ T-SPOT.TB result } \\
\hline Negative & & $118(34.8)$ \\
\hline Positive & & $181(53.4)$ \\
\hline Not done/n & valid result ${ }^{\#}$ & $40(11.8)$ \\
\hline \multicolumn{3}{|c|}{ TST result mm } \\
\hline $5-9$ & & $51(15.0)$ \\
\hline $10-14$ & & $87(25.7)$ \\
\hline$\geqslant 15$ & & $184(54.3)$ \\
\hline Known TST & esponder & $17(5.0)$ \\
\hline
\end{tabular}

Data are presented as n (\%). BCG: bacille Calmette-Guérin; QFT-GIT: QuantiFERON TBв Gold In-tube. \#: no T-SPOT.TBв result because of blood collection failure $(n=12)$, technical failure $(n=4)$, inconclusive test results $(n=5)$ or insufficient blood collected to perform the test $(n=19)$. QFT-GIT is manufactured by Cellesis, Carnegie, Australia; T-SPOT.TB® is manufactured by Oxford Immunotec, Abingdon, UK.

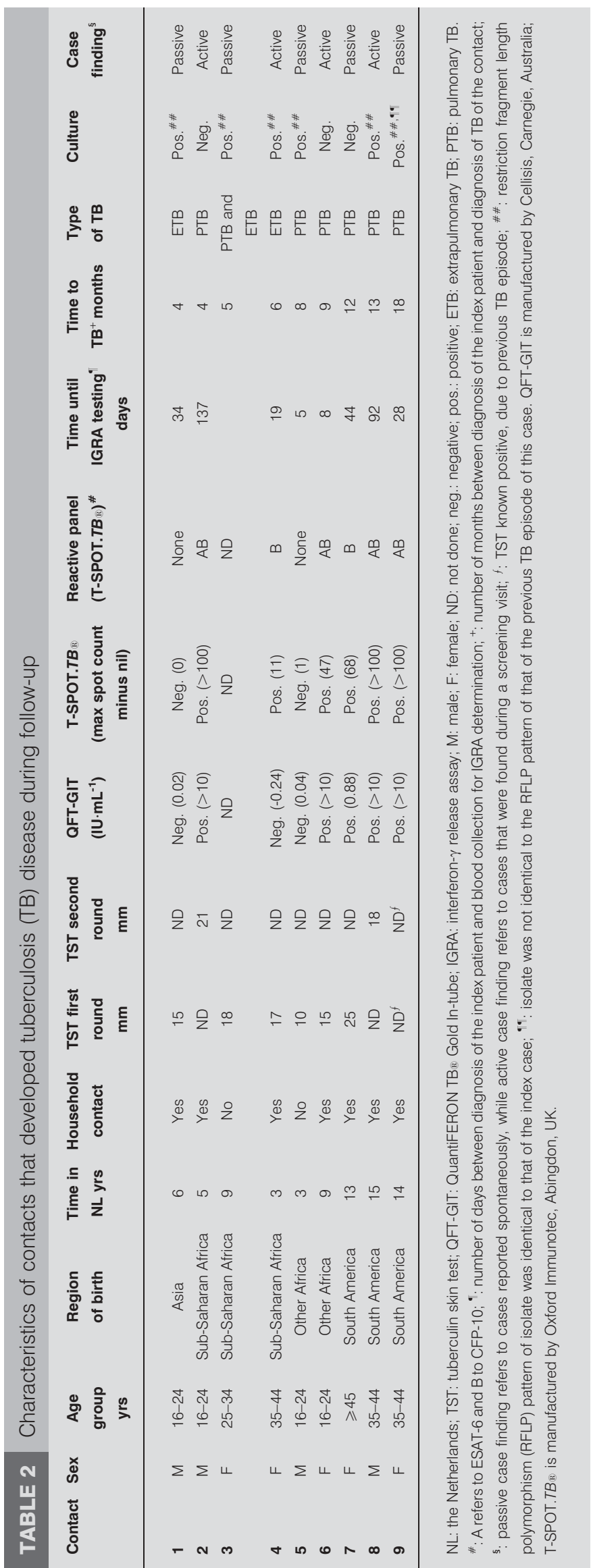


had at least one negative IGRA result were tested relatively early, at 5, 19 (not negative in T-SPOT.TB ${ }$ ) and 34 days after diagnosis of the index patient.

\section{Survival analysis}

No significant difference was observed between the incidence of TB in IGRA-positive and IGRA-negative contacts (QFT-GIT: Gehan-Breslow-Wilcoxon test $\mathrm{p}=0.718$; T-SPOT.TB $B_{\mathbb{R}}: \mathrm{p}=0.443$ ) (fig. 2). Using a cut-off of $15 \mathrm{~mm}$, the difference between the incidence of TB among contacts who were TST positive or negative was not statistically different $(p=0.081)$.

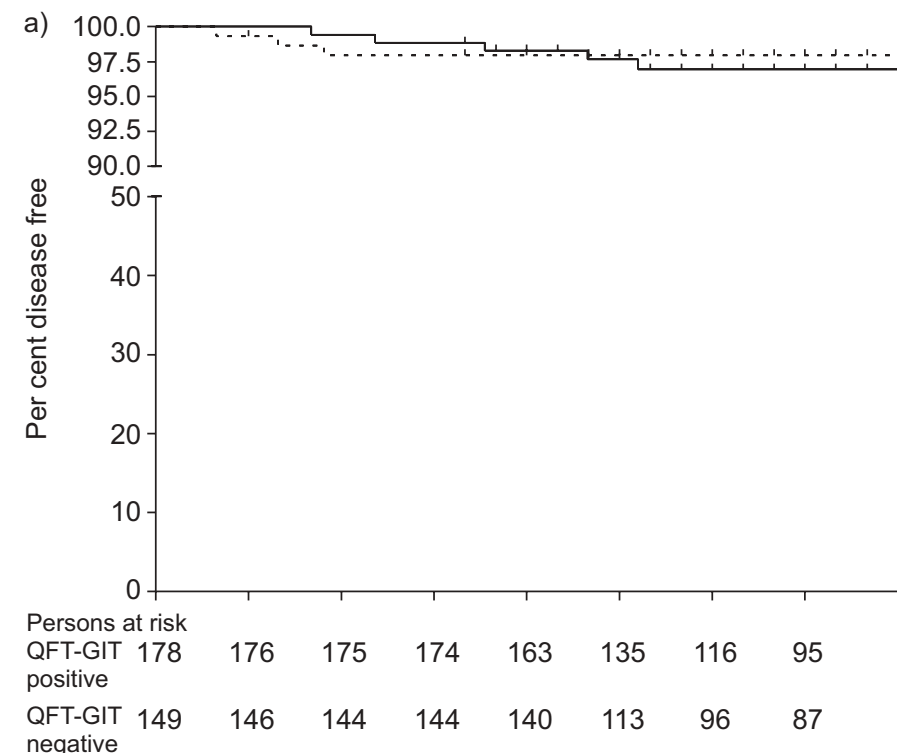

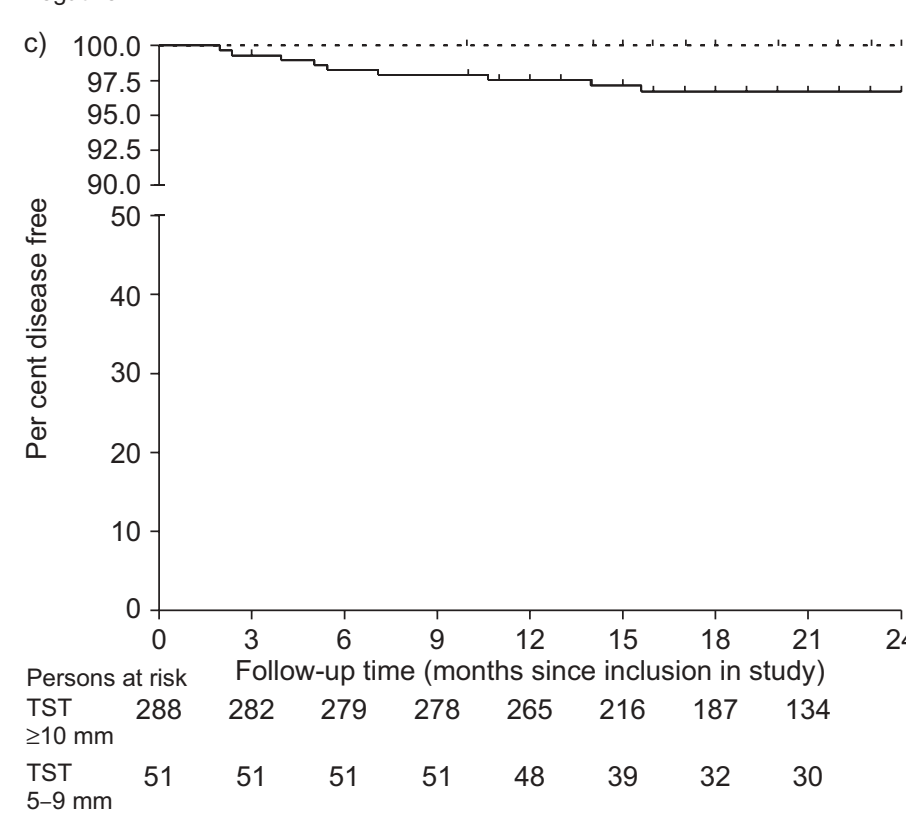

\section{Predictive values}

The 339 contacts were followed for a median (interquartile range) follow-up time of $1.83(1.30-2.00)$ yr. This corresponded with an incidence rate of 16 per 1,000 person-yrs (95\% CI $7.3-$ 30.5). The PPV for progression to TB was 3.1\% (95\% CI $1.3-$ $5.0 \%$ ) for $\mathrm{TST} \geqslant 10 \mathrm{~mm}, 3.8 \%$ (95\% CI $1.7-5.9 \%$ ) for TST $\geqslant 15 \mathrm{~mm}, 2.8 \%$ (95\% CI 1.0-4.6\%) for QFT-GIT and 3.3\% (95\% CI $1.3-5.3 \%$ ) for T-SPOT.TB ${ }$, and sensitivity for TB disease was $100 \%, 88 \%, 63 \%$ and $75 \%$, respectively (table 3 ). Specificity of the tests in this group of contacts with TST $\geqslant 5 \mathrm{~mm}$ (or known positive result), was highest for QFT-GIT (46\%),
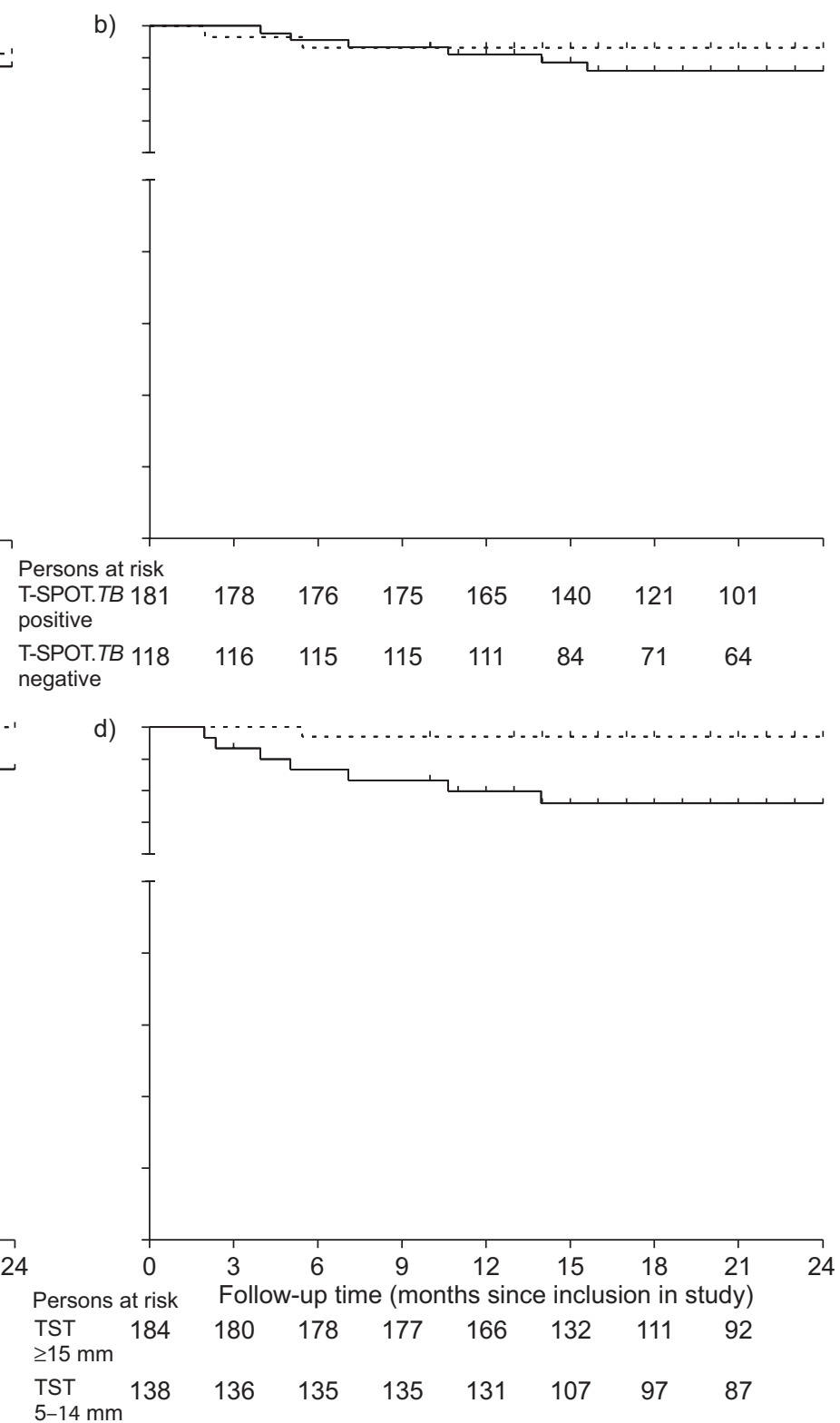

FIGURE 2. Kaplan-Meier curves showing the proportion of tuberculosis (TB)-free contacts with a positive (- $\longrightarrow$ ) or negative $(\cdots \cdots)$ result in a) QuantiFERON-TB $\overline{\mathbb{R}}$ Gold In-tube (QFT-GIT), b) T-SPOT.TB® , c) tuberculin skin test (TST) at a cut-off of $10 \mathrm{~mm}$ and d) TST at a cut-off of $15 \mathrm{~mm}$. Follow-up time was calculated from the date of start of follow-up (the date 3 months after the diagnosis of the index patient, or the date of blood collection for those who had interferon- $\gamma$ release assay testing $>3$ months after the diagnosis of their index patient) up to 24 months, the date of TB diagnosis, the time of emigration or death of the subject, or August 1, 2008, whichever date came first. QFTGIT is manufactured by Cellisis, Carnegie, Australia; T-SPOT.TB $\mathbb{B}_{\mathbb{B}}$ is manufactured by Oxford Immunotec, Abingdon, UK. 
TABLE 3 Sensitivity, specificity and predictive values for development of tuberculosis (TB) disease for QuantiFERON-TB Gold In-tube (QFT-GIT), T-SPOT.TB $B_{\mathbb{B}}$ and tuberculin skin test (TST) among immigrant contacts

\begin{tabular}{|c|c|c|c|c|c|c|c|c|c|c|}
\hline \multirow[t]{2}{*}{ Time point } & \multirow[t]{2}{*}{ Contacts } & \multirow[t]{2}{*}{$\begin{array}{l}\text { Incident TB } \\
\text { cases }\end{array}$} & \multicolumn{2}{|c|}{$\begin{array}{l}\text { Incident TB } \\
\text { cases }\end{array}$} & \multicolumn{2}{|c|}{$\begin{array}{l}\text { Other } \\
\text { contacts }\end{array}$} & \multirow[t]{2}{*}{$\begin{array}{l}\text { Sensitivity } \\
(95 \% \mathrm{Cl})^{5}\end{array}$} & \multirow[t]{2}{*}{$\begin{array}{l}\text { Specificity } \\
(95 \% \text { Cl })^{5}\end{array}$} & \multirow[t]{2}{*}{$\operatorname{PPV}(95 \% \mathrm{Cl})^{\S}$} & \multirow[t]{2}{*}{ NPV $(95 \% \mathrm{Cl})^{\S}$} \\
\hline & & & Test + & Test - & Test + & Test - & & & & \\
\hline \multicolumn{11}{|c|}{ Primary analysis ${ }^{\#}$} \\
\hline $\begin{array}{l}\text { TST } \geqslant 10 \mathrm{~mm} \\
\text { TST } \geqslant 15 \mathrm{~mm} \\
\text { QFT-GIT } \\
\text { T-SPOT.TB } \\
\text { Secondary analy }\end{array}$ & $\begin{array}{l}339 \\
322 \\
327 \\
299\end{array}$ & $\begin{array}{l}9^{f} \\
8^{f} \\
8 \\
8\end{array}$ & $\begin{array}{l}9 \\
7 \\
5 \\
6\end{array}$ & $\begin{array}{l}0 \\
1 \\
3 \\
2\end{array}$ & $\begin{array}{l}279 \\
177 \\
173 \\
175\end{array}$ & $\begin{array}{c}51 \\
137 \\
146 \\
116\end{array}$ & $\begin{array}{c}100(100-100) \\
88(84-91) \\
63(57-68) \\
75(70-80)\end{array}$ & $\begin{array}{l}15(12-19) \\
44(38-49) \\
46(40-51) \\
40(34-45)\end{array}$ & $\begin{array}{l}3.1(1.3-5.0) \\
3.8(1.7-5.9) \\
2.8(1.0-4.6) \\
3.3(1.3-5.3)\end{array}$ & $\begin{array}{r}100(100-100) \\
99.3(98.4-100) \\
98.0(96.5-99.5) \\
98.3(96.8-99.8)\end{array}$ \\
\hline $\begin{array}{l}\text { TST } \geqslant 10 \mathrm{~mm} \\
\text { TST } \geqslant 15 \mathrm{~mm} \\
\text { QFT-GIT } \\
\text { T-SPOT.TB } \\
\text { Sensitivity analy }\end{array}$ & $\begin{array}{l}334 \\
317 \\
323 \\
295\end{array}$ & $\begin{array}{c}7 \\
7 \\
6^{\# \#} \\
6^{\# \#}\end{array}$ & $\begin{array}{l}7 \\
6 \\
3 \\
4\end{array}$ & $\begin{array}{l}0 \\
1 \\
3 \\
2\end{array}$ & $\begin{array}{l}278 \\
176 \\
173 \\
175\end{array}$ & $\begin{array}{c}49 \\
134 \\
144 \\
114\end{array}$ & $\begin{array}{c}100(100-100) \\
86(82-90) \\
50(45-55) \\
67(61-72)\end{array}$ & $\begin{array}{l}15(11-19) \\
43(38-49) \\
45(40-51) \\
39(34-45)\end{array}$ & $\begin{array}{l}2.5(0.8-4.1) \\
3.3(1.3-5.3) \\
1.7(0.3-3.1) \\
2.2(0.5-3.9)\end{array}$ & $\begin{array}{r}100(100-100) \\
99.3(98.3-100) \\
98.0(96.4-99.5) \\
98.3(96.8-99.8)\end{array}$ \\
\hline $\begin{array}{l}\text { TST } \geqslant 10 \mathrm{~mm} \\
\text { TST } \geqslant 15 \mathrm{~mm} \\
\text { QFT-GIT } \\
\text { T-SPOT.TB }{ }_{\mathbb{R}}\end{array}$ & $\begin{array}{l}203 \\
191 \\
201 \\
186\end{array}$ & $\begin{array}{c}7 \\
7 \\
6^{\# \#} \\
6^{\# \#}\end{array}$ & $\begin{array}{l}7 \\
6 \\
3 \\
4\end{array}$ & $\begin{array}{l}0 \\
1 \\
3 \\
2\end{array}$ & $\begin{array}{l}165 \\
107 \\
112 \\
114\end{array}$ & $\begin{array}{l}31 \\
77 \\
83 \\
66\end{array}$ & $\begin{array}{c}100(100-100) \\
86(81-91) \\
50(43-57) \\
67(60-73)\end{array}$ & $\begin{array}{l}16(11-21) \\
42(35-49) \\
43(36-49) \\
37(30-44)\end{array}$ & $\begin{array}{l}4.1(1.4-6.8) \\
5.3(2.1-8.5) \\
2.6(0.4-4.8) \\
3.4(0.8-6.0)\end{array}$ & $\begin{array}{r}100(100-100) \\
98.7(97.1-100) \\
96.5(94.0-99.1) \\
97.1(94.6-99.5)\end{array}$ \\
\hline
\end{tabular}

Data are presented as $n$, unless otherwise stated. +: positive; -: negative; PPV: positive predictive value; NPV: negative predictive value. ${ }^{*}$ : all contacts with TST $\geqslant 5$ mm; test parameters determined with follow-up until August 1, 2008. ": contacts with TST $\geqslant 5 \mathrm{~mm}$, who started follow-up $\geqslant 12$ months before August 1, 2008; test parameters are determined after 12 months follow-up. ${ }^{+}$: contacts with TST $\geqslant 5 \mathrm{~mm}$, who were actively followed for $\geqslant 12$ months; test parameters determined after 12 months of follow-up. ${ }^{\text {s: }}$ sensitivity, specificity, PPV and NPV for development of TB disease are determined in immigrant close contacts with TST $\geqslant 5 \mathrm{~mm}$. ${ }^{f}$. TST was known positive ( $\left.\geqslant 10 \mathrm{~mm}\right)$ in one incident TB case and not

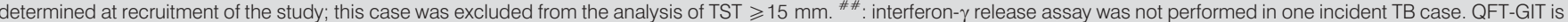
manufactured by Cellesis, Carnegie, Australia; T-SPOT.TBæ is manufactured by Oxford Immunotec, Abingdon, UK.

followed by TST (cut-off $15 \mathrm{~mm})(44 \%)$, T-SPOT.TBß $(40 \%)$ and lowest for TST (cut-off $10 \mathrm{~mm})(15 \%)$.

Five contacts were excluded in the secondary analysis, since their follow-up started $<12$ months before August 1, 2008. The incidence rate during the first 12 months was 21 per 1,000 person-yrs (95\% CI 8.6-44.2). The PPV in the first 12 months was again not better for QFT-GIT $(1.7 \%, 95 \%$ CI $0.3-3.1 \%)$ or T-SPOT.TB $B_{\circledast}(2.2 \%, 95 \%$ CI $0.6-3.9 \%)$ than for the TST using a cut-off of $10 \mathrm{~mm}(2.5 \%, 95 \%$ CI $0.8-4.1 \%)$ or $15 \mathrm{~mm}(3.3 \%, 95 \%$ CI $1.4-5.2 \%$ )

Restricting the analysis to contacts who attended the follow-up visits, the IGRA did not have a higher PPV compared to the TST either at a cut-off of 10 or $15 \mathrm{~mm}$, although all PPVs were slightly increased.

\section{DISCUSSION}

In this prospective cohort study including recently exposed immigrant close contacts with TST results $\geqslant 5 \mathrm{~mm}$ who were followed without preventive treatment, we found that the PPVs of QFT-GIT and T-SPOT.TB for subsequent development of TB disease during the first 2 yrs after a contact investigation were comparable to that of the TST, irrespective of the TST cutoff $(10$ or $15 \mathrm{~mm})$. Our results differ from those in other populations in low-incidence settings [9] that showed that the QFT-GIT may be a good predictor for development of active TB. In our study, over half of the tested immigrant close contacts were QFT-GIT or T-SPOT.TB ${ }_{\mathbb{B}}$ positive. When we assume that contacts with TST $<5 \mathrm{~mm}$, whom we had excluded from IGRA testing, would have been IGRA negative, still 42 $46 \%$ of the contacts would be IGRA positive. This high proportion of positive tests found among recently exposed immigrant contacts is probably not only attributable to recently acquired infections [13].
So far, five other contact studies assessed progression to disease in contacts tested with an IGRA [8-12] in different populations. DiEL et al. [9] found six TB patients among 41 QFT-GIT positive contacts. The PPV of the QFT-GIT in this study $(14.6 \%)$ was significantly higher than when a TST cut-off of $5 \mathrm{~mm}$ was used (PPV 2.3\%; $\mathrm{p}=<0.003$ ), although not at a cut-off of $10 \mathrm{~mm}$ (PPV 5.6\%; $\mathrm{p}=0.10$ ). In contrast, two studies assessing the ELISPOT in household contacts in Gambia [11] or in child contacts in Turkey [8] reported a similar prediction of TB cases by ELISPOT compared with the TST. Similar to our findings, in the latter two studies $[8,11]$ the IGRA missed some of the contacts who progressed to disease. It is unclear if discrepancies between these studies may be explained by differences in the type of IGRA that was used. Direct comparison between the QFT-GIT and T-SPOT.TB ${ }_{\circledR}$ showed that discrepancies occur, and T-SPOT.TB $B_{\mathbb{R}}$ seemed to be slightly more sensitive than QFT-GIT [13, 18-21]. Probably of more importance are the differences in the populations studied and the TB incidence in these countries.

In contrast to our expectations, three incident cases had a negative IGRA result. Re-infection and comorbidity were unlikely explanations for the negative IGRA results. We performed the IGRA only once and usually shortly after the diagnosis of the index patient. Although the time to positivity after infection may be shorter for IGRA than for the TST [22], it is possible that we tested our contacts too early and the IGRA was not yet positive in contacts who later progressed to disease. On the contrary, reversions of previously positive IGRA results have also been reported [23, 24]. More studies are needed to determine the optimal moment for IGRA testing after infection to develop new diagnostic algorithms for LTBI.

While the immigrant contacts in our study were all recently exposed, we observed previously that positive IGRA results 
may also be associated with remote infection [13]. The implementation of IGRAs in clinical practice in high TB endemic settings or among individuals with a high likelihood of previous exposure, as recommended by some [25], is therefore debatable [26, 27]. Nevertheless, the incidence rate among the recently exposed immigrant contacts was relatively high (16 per 1,000 person-yrs) compared to estimations of others who assessed close contacts, ranging 3.2-12.5 per 1,000 person-yrs [8, 11, 28, 29]. Based on these results it may be recommended to incorporate the use of preventive therapy or other preventive measures in the Dutch setting for the screening of immigrant close contacts of sputum smearpositive TB cases, as is already the practice in many other low-incidence countries [3-5,30]. The choice of the diagnostic test to be used may be based on their cost-effectiveness.

Our study had some shortcomings. First, we determined the IGRA and followed contacts actively only when TST $\geqslant 5 \mathrm{~mm}$. The exclusion of contacts with TST results $<5 \mathrm{~mm}$ may have influenced our PPV and sensitivity estimations only to a limited extent. Few contact studies reported the percentage of positive IGRA results among contacts with TST $<5 \mathrm{~mm}$, but found this to be $<10 \%[9,18,19,31,32]$. Moreover, their risk of progression to disease is negligible [33] and we did not observe any case of TB in this subgroup upon checking the NTR. When one would assume that none of the immigrants with TST $<5 \mathrm{~mm}$ would be IGRA positive, and indeed no TB cases occurred in this group, by definition this would result in the same PPV and sensitivity as estimated here. However, we may have underestimated the specificity and NPV when tests would be used directly as a single test (instead of after TST $\geqslant 5 \mathrm{~mm}$ ). Secondly, we did not have complete follow-up data for all contacts. Although it is likely that contacts who stopped attending the follow-up visits were less likely to have developed TB disease, since they would otherwise have been notified to the NTR, we do not know this with certainty. Nevertheless, when the analysis was restricted to contacts followed for $\geqslant 12$ months, we found the same pattern of PPVs as in our primary analysis, implying that non-participation in the follow-up visits will have had limited effect on our findings. Furthermore, our sample size was too small to determine superiority of one of the tests over the others. More longitudinal studies are needed to reveal which test will predict disease progression best, and in which persons.

In conclusion, we observed a high incidence rate of TB disease among immigrant close contacts during the subsequent 2 yrs of follow-up. The PPV for progression to TB among immigrant close contacts of both IGRAs was not better than that of the TST. The incidence found among the study population justifies active preventive measures in this group.

\section{SUPPORT STATEMENT}

This study was funded by unrestricted grants from the Netherlands Organization for Health Research and Development (ZonMw; the Hague, the Netherlands).

\section{STATEMENT OF INTEREST}

A statement of interest for this study can be found at www.erj. ersjournals.com/misc/statements.dtl

\section{ACKNOWLEDGEMENTS}

The authors wish to thank all the participants and the staff of the participating municipal health services: GGD Amsterdam, GGD Den Haag, GGD Eindhoven, Hulpverleningsdienst Flevoland, Hulpverlening Gelderland Midden, Hulpverleningsdienst GGD Groningen (locations Groningen and Assen), GGD Hart voor Brabant, GGD Hollands Midden, GGD Regio Nijmegen, GGD Rotterdam e.o., GGD Regio Twente, GGD Utrecht, GGD West-Brabant, GGD Zuid-Holland West and GGD Zuidoost-Brabant, and H. el Bannoudi (Dept of Infectious Diseases, Leiden University Medical Centre, Leiden, the Netherlands) for technical assistance at the laboratory.

\section{APPENDIX}

List of birth countries considered not to be high endemic for this study: Australia, Austria, Belgium, Canada, Czech Republic, Cyprus, Denmark, Estonia, Finland, Germany, Greece, Hungary, Iceland, Ireland, Israel, Italy, Japan, Latvia, Lithuania, Luxembourg, Malta, Monaco, New Zealand, Norway, Poland, Portugal, Slovakia, Slovenia, Spain, Sweden, Suriname (if the individual has not received a BCG vaccination in Suriname during childhood), Switzerland, UK and USA.

\section{REFERENCES}

1 Menzies D, Pai M, Comstock G. Meta-analysis: new tests for the diagnosis of latent tuberculosis infection: areas of uncertainty and recommendations for research. Ann Intern Med 2007; 146: 340-354.

2 Menzies D. Interpretation of repeated tuberculin tests. Boosting, conversion, and reversion. Am J Respir Crit Care Med 1999; 159: 15-21.

3 NIHES. Tuberculosis. Clinical diagnosis and management of tuberculosis, and measures for its prevention and control. Clinical guideline 33: National Institute for Health and Clinical Excellence 2006; 1-66.

4 Pai M, Gardam M, Haldane D, et al. An Advisory Committee Statement. Canadian Tuberculosis Committee. Updated recommendations on interferon gamma release assays for latent tuberculosis infection. CCDR 2008; 34: 1-13.

5 Diel R, Foreßbohm M, Loytved G, et al. Empfehlungen für die Umgebungsuntersuchungen bei Tuberkulose - Deutsches Zentralkomitee zur Bekämpfung der Tuberkulose [Recommendations for background studies in tuberculosis]. Pneumologie 2007; 61: 440-455.

6 Mazurek GH, Jereb J, Lobue P, et al. Guidelines for using the QuantiFERON-TB Gold test for detecting Mycobacterium tuberculosis infection, United States. MMWR Recomm Rep 2005; 54: 49-55.

7 Andersen P, Doherty TM, Pai M, et al. The prognosis of latent tuberculosis: can disease be predicted? Trends Mol Med 2007; 13: 175-182.

8 Bakir M, Millington KA, Soysal A, et al. Prognostic value of a T-cell-based, interferon- $\gamma$ biomarker in children with tuberculosis contact. Ann Intern Med 2008; 149: 777-786.

9 Diel R, Loddenkemper R, Meywald-Walter K, et al. Predictive value of a whole blood IFN- $\gamma$ assay for the development of active tuberculosis disease after recent infection with Mycobacterium tuberculosis. Am J Respir Crit Care Med 2008; 177: 1164-1170.

10 Doherty TM, Demissie A, Olobo J, et al. Immune responses to the Mycobacterium tuberculosis-specific antigen ESAT-6 signal subclinical infection among contacts of tuberculosis patients. J Clin Microbiol 2002; 40: 704-706.

11 Hill PC, Jackson-Sillah DJ, Fox A, et al. Incidence of tuberculosis and the predictive value of ELISPOT and Mantoux tests in Gambian case contacts. PLoS One 2008; 3: e1379. 
12 Aichelburg MC, Rieger A, Breitenecker F, et al. Detection and prediction of active tuberculosis disease by a whole-blood interferon- $\gamma$ release assay in HIV-1-infected individuals. Clin Infect Dis 2009; 48: 954-962.

13 Kik SV, Franken WP, Arend SM, et al. Interferon- $\gamma$ release assays in immigrant contacts and effect of remote exposure to Mycobacterium tuberculosis. Int J Tuberc Lung Dis 2009; 13: 820-828.

14 Oxford Immunotec. www.oxfordimmunotec.com/Oxford International. Date last accesssed: June 21, 2009.

15 Cellestis. QuantiFERON TB Gold (In-tube Method). www.cellestis. com/IRM/Company/ShowPage.aspx?CPID $=1170$ Last updated: January 2007. Date last accessed: June 21, 2009.

16 van Embden JD, Cave MD, Crawford JT, et al. Strain identification of Mycobacterium tuberculosis by DNA fingerprinting: recommendations for a standardized methodology. J Clin Microbiol 1993; 31 : 406-409.

17 van Soolingen D, de Haas PE, Hermans PW, et al. Comparison of various repetitive DNA elements as genetic markers for strain differentiation and epidemiology of Mycobacterium tuberculosis. J Clin Microbiol 1993; 31: 1987-1995.

18 Arend SM, Thijsen SF, Leyten EM, et al. Comparison of two interferon- $\gamma$ assays and tuberculin skin test for tracing tuberculosis contacts. Am J Respir Crit Care Med 2007; 175: 618-627.

19 Connell TG, Ritz N, Paxton GA, et al. A three-way comparison of tuberculin skin testing, QuantiFERON-TB gold and T-SPOT.TB in children. PLoS One 2008; 3: e2624.

20 Ferrara G, Losi M, D'Amico R, et al. Use in routine clinical practice of two commercial blood tests for diagnosis of infection with Mycobacterium tuberculosis: a prospective study. Lancet 2006; 367: 1328-1334.

21 Pai M, Zwerling A, Menzies D. Systematic review: T-cell-based assays for the diagnosis of latent tuberculosis infection: an update. Ann Intern Med 2008; 149: 177-184.

22 Franken WP, Koster BF, Bossink AW, et al. Follow-up study of tuberculosis-exposed supermarket customers with negative tuberculin skin test results in association with positive $\gamma$ interferon release assay results. Clin Vaccine Immunol 2007; 14: 1239-1241.
23 Franken WP, Arend SM, Thijsen SF, et al. Interferon- $\gamma$ release assays during follow-up of tuberculin skin test-positive contacts. Int J Tuberc Lung Dis 2008; 12: 1286-1294.

24 Pai M, Joshi R, Dogra S, et al. T-cell assay conversions and reversions among household contacts of tuberculosis patients in rural India. Int J Tuberc Lung Dis 2009; 13: 84-92.

25 Nienhaus A, Schablon A, Diel R. Interferon- $\gamma$ release assay for the diagnosis of latent TB infection - analysis of discordant results, when compared to the tuberculin skin test. PLoS One 2008; 3: e2665.

26 Barth RE, Mudrikova T, Hoepelman AI. Interferon- $\gamma$ release assays (IGRAs) in high-endemic settings: could they play a role in optimizing global TB diagnostics? Evaluating the possibilities of using IGRAs to diagnose active TB in a rural African setting. Int $J$ Infect Dis 2008; 12: e1-e6.

27 Menzies D. Using tests for latent tuberculous infection to diagnose active tuberculosis: can we eat our cake and have it too? Ann Intern Med 2008; 148: 398-399.

28 Guwatudde D, Nakakeeto M, Jones-Lopez EC, et al. Tuberculosis in household contacts of infectious cases in Kampala, Uganda. Am J Epidemiol 2003; 158: 887-898.

29 Lee JY, Choi HJ, Park I-N, et al. Comparison of two commercial interferon- $\gamma$ assays for diagnosing Mycobacterium tuberculosis infection. Eur Respir J 2006; 28: 24-30.

30 Targeted tuberculin testing and treatment of latent tuberculosis infection. American Thoracic Society. MMWR Recomm Rep 2000; 49: 1-51.

31 Brock I, Weldingh K, Lillebaek T, et al. Comparison of tuberculin skin test and new specific blood test in tuberculosis contacts. Am J Respir Crit Care Med 2004; 170: 65-69.

32 Janssens JP, Roux-Lombard P, Perneger T, et al. Contribution of a IFN- $\gamma$ assay in contact tracing for tuberculosis in a lowincidence, high immigration area. Swiss Med Wkly 2008; 138: 585-593.

33 Comstock GW, Livesay VT, Woolpert SF. The prognosis of a positive tuberculin reaction in childhood and adolescence. Am J Epidemiol 1974; 99: 131-138. 